\title{
Modification on interval symmetric single-step procedure ISS-5 $\delta$ for bounding polynomial zeros simultaneously
}

\begin{abstract}
The purpose of this paper is to establish a new modified method. This modified procedure is called the Interval Symmetric Single Step-5 Delta Procedure ISS-5ŭ This research start with some disjoints intervals as the initial intervals which contain the polynomial zeros. The procedure of ISS-5ŭ will generate smaller bounded close intervals. The procedure is run on 5 test polynomials and the results obtained show that this procedure is more efficient than previous procedure.
\end{abstract}

Keyword: Analysis interval; Determination of polynomial zeros; Interval procedure; Simultaneous inclusion 